\title{
Dark Matter Searches with the ATLAS detector
}

\author{
Alison Elliot ${ }^{1, \star}$ on behalf of the ATLAS Collaboration \\ ${ }^{1}$ University of Victoria
}

\begin{abstract}
The presence of a non-baryonic dark matter component in the Universe is inferred from the observation of its gravitational interaction. If dark matter interacts weakly with the Standard Model it would be produced at the LHC, escaping the detector and leaving a large missing transverse momentum as its signature. The ATLAS detector has developed a broad and systematic search program for dark matter production in LHC collisions. The results of these searches on the first $13 \mathrm{TeV}$ data, their interpretation, and the design and possible evolution of the search program will be presented.
\end{abstract}

\section{Introduction}

Dark Matter is an unsolved problem; there is nothing in the Standard Model that can explain it. However, something beyond this theory clearly exists. The astrophysical indicators of Dark Matter (DM) come primarily from the cosmic microwave background [1, 2], gravitational lensing [3], the motion of stars in galaxies [4], and the motion of galaxies in clusters [5].

In order to discover the nature of DM, some assumptions must be made about it. The most important assumption is that DM is a particle that interacts weakly with the Standard Model (SM) [6, 7]. Once this assumption is made, DM can be searched for in emission from galactic sources, from direct detection in nuclear recoils underground, or from particle production in colliders. The searches being performed using the $13 \mathrm{TeV}$ dataset from the ATLAS detector [8] at the CERN Large Hadron Collider (LHC) [9] will be presented here.

\section{The ATLAS Dataset}

The data taken at the ATLAS detector at $13 \mathrm{TeV}$ during 2015 and 2016 are used for the following searches. The recorded luminosity during these time periods can be seen in Figure 1. The searches presented use either the 2015 dataset with $3.2 \mathrm{fb}^{-1}$, the full $2015+2016$ dataset with $36.1 \mathrm{fb}^{-1}$, or a 2015 plus a subset of 2016 data, amounting to $13.3 \mathrm{fb}^{-1}$.

\section{Dark Matter Searches}

The LHC DM Working Group is a collaboration between ATLAS, CMS, and the theory community in order to benchmark interesting models in the search for DM. The working group's recommendations

\footnotetext{
^e-mail: alison.elliot@cern.ch
} 

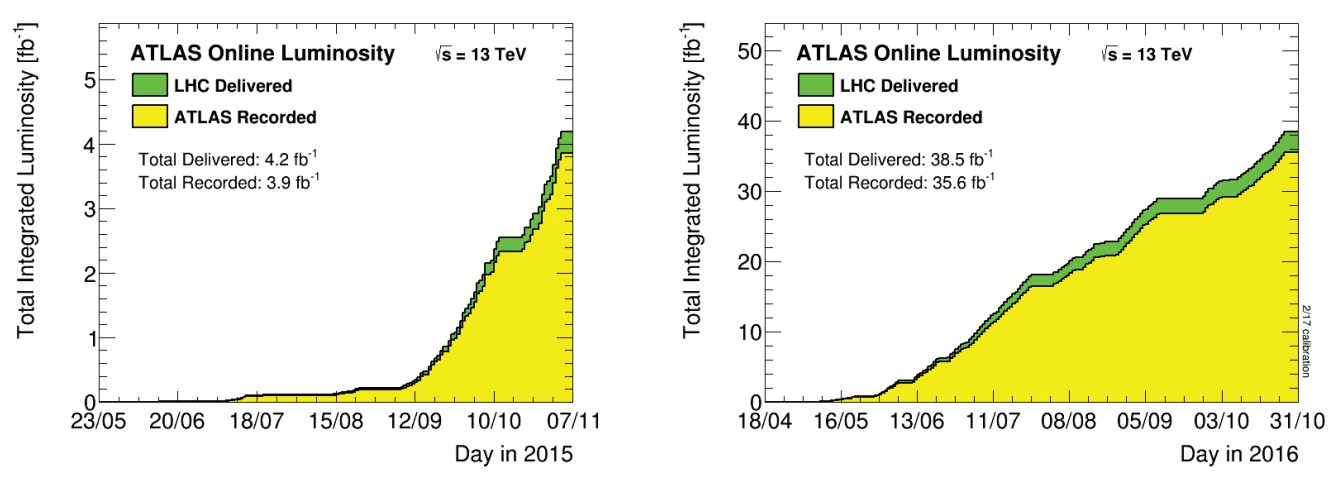

Figure 1. ATLAS recorded luminosity at $13 \mathrm{TeV}$ by day in 2015 (left) and 2016 (right).

for the $13 \mathrm{TeV}$ data collected at the LHC are found in Ref. [10]. These benchmark models include effective field theory (EFT) models which are defined by their interaction mass or energy scale $\left(M^{*}\right.$ or $\Lambda$ ). Other models included are the simple mediator models, often referred to as 'simplified models', whose parameters include the mass of the mediator $\left(m_{\mathrm{med}}\right)$ and the mass of the DM $\left(m_{\chi}\right)$. Both EFT and simplified models are measurable in the ATLAS detector when the DM particles are produced in conjunction with SM particles, which arise either as initial state radiation (ISR), or through direct contact at the interaction.

Further DM models being considered are 'two Higgs doublet models'. These models include either vector $\left(Z^{\prime}\right)$ or scalar $\left(h, \mathrm{~A}^{0}, S\right)$ particles that act as mediators to DM, as well as SM particles that interact in the detector. Recommendations and benchmarks for these models can also be found in Ref. [10].

\subsection{Mono-X Searches}

Searches for DM plus a detectable SM particle are referred to as 'Mono- $\mathrm{X}$ ' searches, where ' $\mathrm{X}$ ' is the SM particle being produced in conjunction with the DM particle(s). In Mono-X searches, the presence of DM is inferred from a momentum imbalance in the ATLAS detector. The key variable to these searches is the magnitude of the missing momentum, referred to as missing energy, which is in the plane transverse to the beam direction. The symbol for this variable is $E_{\mathrm{T}}^{\mathrm{miss}}$. The amount of $E_{\mathrm{T}}^{\mathrm{miss}}$ expected from the DM depends on the masses of both the DM and its mediator.

Other important variables and selections applied to the potential DM events in Mono-X include suppression of fake $E_{\mathrm{T}}^{\text {miss }}$ through proxy on its uncertainty $\left(E_{\mathrm{T}}^{\text {miss }} / \sqrt{\Sigma E_{\mathrm{T}}}\right)$ and angular requirements, which could include a large separation $\Delta \phi$ between the missing momentum and the SM particle momentum or separation between the missing momentum and hadronic activity in the detector.

\section{Mono-photon}

One of the cleanest Mono-X signatures of new physics is the Mono- $\gamma$ search. In this search, using $36.1 \mathrm{fb}^{-1}$ of data collected at ATLAS, an ISR $\gamma$ is produced along with at least $150 \mathrm{GeV}$ of $E_{\mathrm{T}}^{\text {miss }}$ [11], see Figure 2 (left). Events with leptons or more than one jet are vetoed. The background is predominantly $Z+$ jets, where $Z \rightarrow v \bar{v}$. Normalization factors for the backgrounds are obtained from a simultaneous background-only fit. 

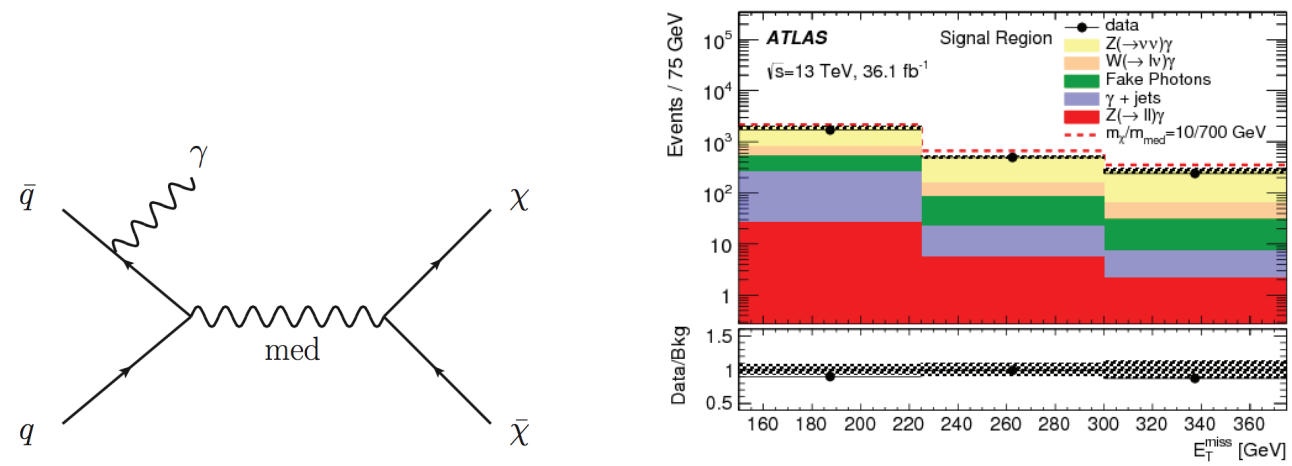

Figure 2. The Feynman diagram for a simplified model of dark matter with an ISR $\gamma$ (left). The number of events in the backgrounds to the search as well as the data measurement are shown in bins of $E_{\mathrm{T}}^{\text {miss }}$ up to $400 \mathrm{GeV}$ (right) [11].

Using the results in Figure 2 (right), limits can be placed on the simplified models of DM that were analyzed. As seen in Figure 3, mediator masses up to $1.2 \mathrm{TeV}$ can be excluded for the axialvector mediator simplified model. As well, limits on the DM-nucleon cross-section for interaction are extremely competitive with direct detection searches at low and mid-range DM masses.
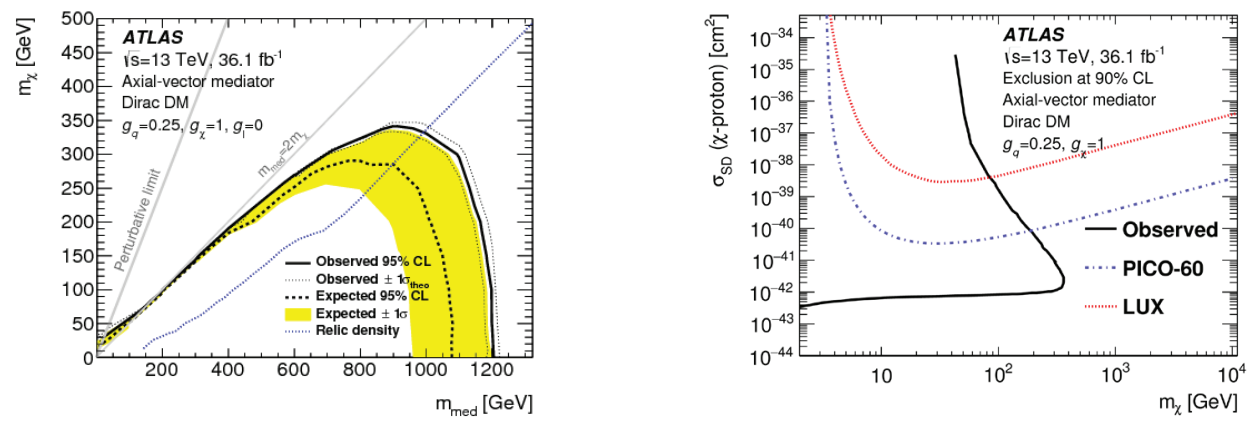

Figure 3. The 95\% exclusion contours for axial-vector mediated simplified Mono- $\gamma$ models of DM, in mass of the mediator, $m_{\text {med }}$, and mass of the DM, $m_{\chi}$ (left). Exclusion contours at $90 \%$ CL reinterpreted as spin-dependent DM-nucleon coupling strengths for various mediator masses, and compared to direct detection searches (right) [11].

\section{Mono-jet}

One of the first places Mono-X searches could find DM is the Mono-jet search [12], as ISR jets are a frequent occurrence and a distinct signature in the detector. In this search, using $3.2 \mathrm{fb}^{-1}$ of data, an energetic ISR jet is produced along with at least $250 \mathrm{GeV}$ of $E_{\mathrm{T}}^{\text {miss }}$. An angular distance separation is applied to ensure the jets and $E_{\mathrm{T}}^{\text {miss }}$ are well-separated, and any charged leptons are vetoed. The backgrounds, such as $Z \rightarrow v \bar{v}+$ jets, are normalized to data through Monte Carlo (MC) control regions. The results exclude the axial-vector mediator simplified model of $\mathrm{DM}$ out to $1 \mathrm{TeV}$, on 
masses of DM over $250 \mathrm{GeV}$. Cross section limits are competitive with the direct detection searches at low and mid-ranged DM masses, as seen in Figure 4.
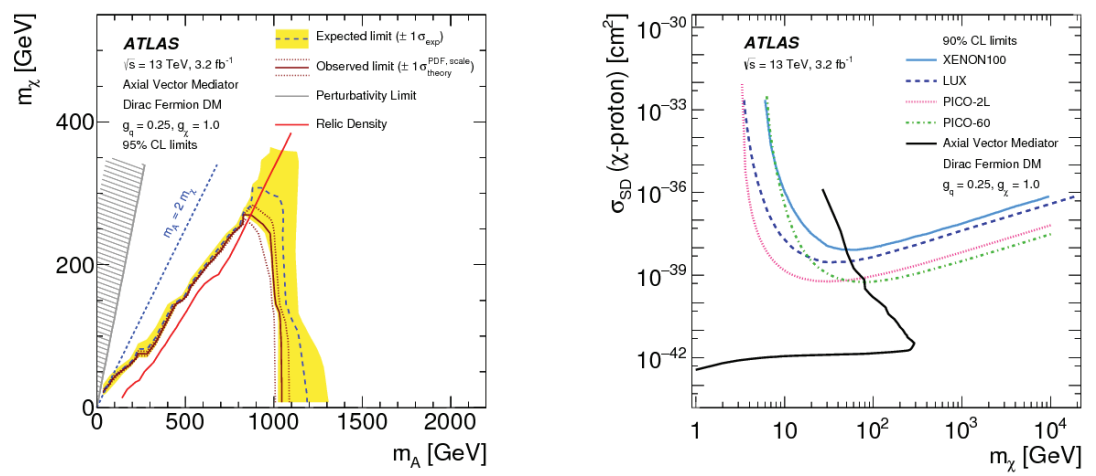

Figure 4. The 95\% exclusion contours for axial-vector mediated simplified Mono-jet models of DM, by mass of the mediator, $m_{\text {med }}$, and mass of the DM, $m_{\chi}$ (left). Exclusion contours at $90 \% \mathrm{CL}$ reinterpreted as spin-dependent DM-nucleon coupling strengths for various mediator masses and compared to direct detection searches (right) [12].

\section{Mono-V}

A search similar to the Mono-jet search is the Mono-V(hadronic) search [13]. This search for DM examines $3.2 \mathrm{fb}^{-1}$ of data for an ISR $W$ or $Z$ boson in the form of an energetic jet containing substructure consistent with coming from a $W$ or $Z$ boson and at least $250 \mathrm{GeV}$ of missing energy. Backgrounds, such as $Z \rightarrow v \bar{v}+$ jets, are estimated through MC and normalized to data through dedicated control regions. Both vector-mediated simplified models as well as EFT contact models are considered, and limits are set on the signal strength and couplings respectively. The EFT Feynman diagram and results can be seen in Figure 5.
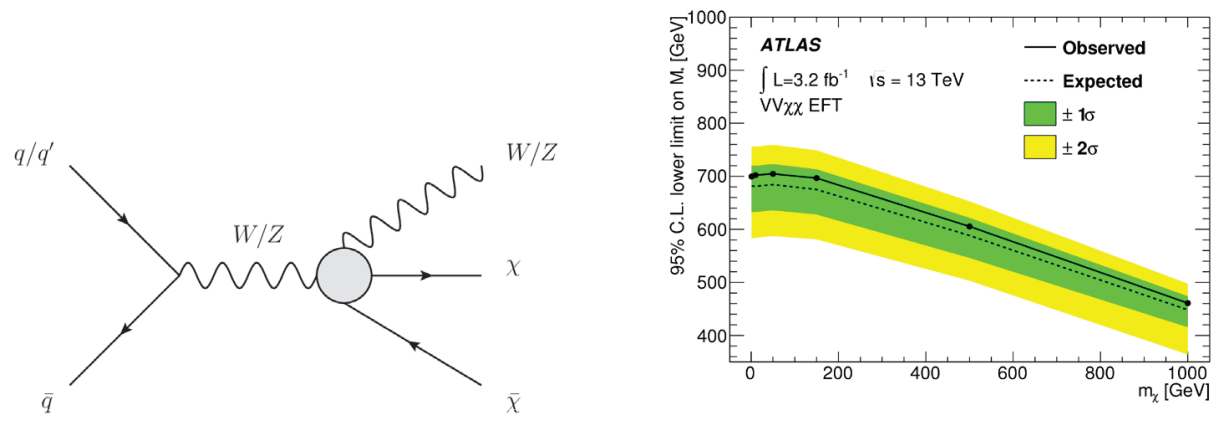

Figure 5. The Feynman diagram for an EFT model of dark matter in a contact interaction with a $Z$ boson (left). 95\% CL lower limits on EFT effective mass for various dark matter masses $m_{\chi}$ (right) [13]. 
An analysis with a similar structure is the Mono- $Z(\ell \ell)$ search that is performed with $13.3 \mathrm{fb}^{-1}$ looking for the emission of an ISR $Z$ boson where the $Z$ boson decays into charged leptons [14]. This process has a smaller cross section (from the smaller branching fraction of the $Z \rightarrow \ell \ell$ ), but a cleaner signal than the Mono-V search. The background arises primarily from diboson processes, as well as $Z \rightarrow \ell \ell+$ jets, and is estimated through MC and normalized by dedicated control regions, see Figure 6.
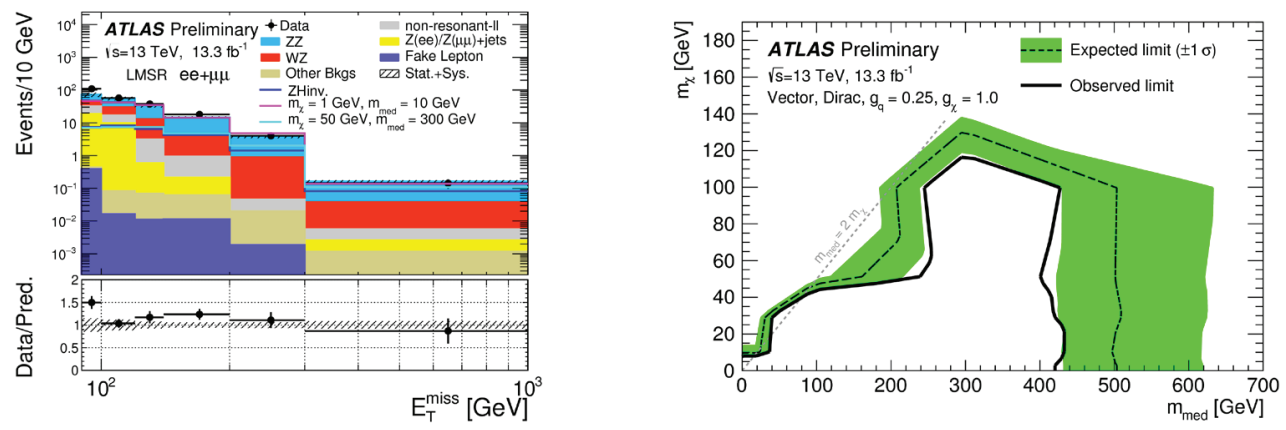

Figure 6. The number of events in the backgrounds to the Mono- $Z(\ell \ell)$ search as well as the data measurement are shown in bins of $E_{\mathrm{T}}^{\text {miss }}$ up to $1000 \mathrm{GeV}$ (left). The $95 \%$ exclusion contours for axial-vector mediated simplified models of DM, by mass of the mediator, $m_{\mathrm{med}}$ and mass of the DM, $m_{\chi}$ (right) [14].

\section{Mono-H}

Another suite of analyses that have sensitivities to different models of DM are the Mono-H searches. They search for two Higgs doublet models, such as the one picture in Figure 7 (left). There are several searches for DM that are separated by the decay of the Higgs boson in the model. One such search is the Mono- $\mathrm{H}(b b)$ with $36.1 \mathrm{fb}^{-1}$ of data [15], where the large branching fraction of the Higgs to $b$-quarks is exploited. The backgrounds, such as $Z \rightarrow v \bar{v}$ and $t$-quark backgrounds, are estimated through control regions in data. Limits are made on the mass of a $Z^{\prime}$ mediator up to $2.5 \mathrm{TeV}$, for pseudo scalar masses $\left(m_{A}\right)$ up to $600 \mathrm{GeV}$, as seen in Figure 7 (right).
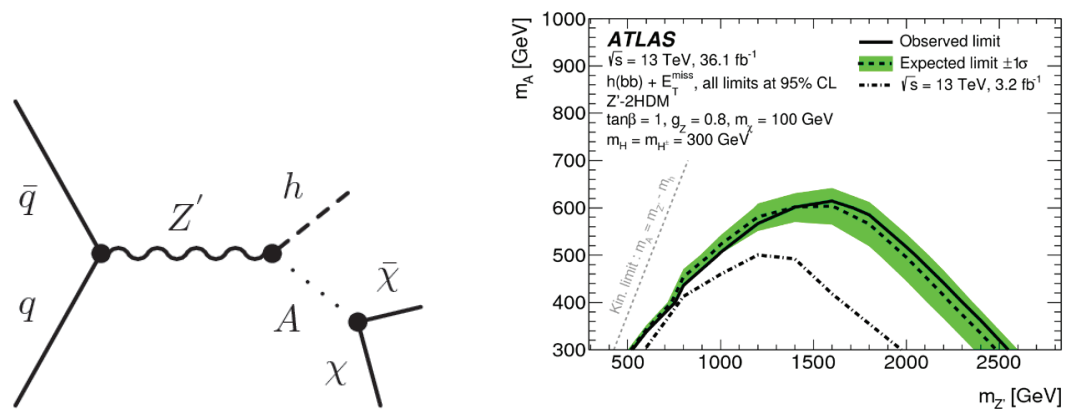

Figure 7. The Feynman diagram for a Mono-H model of dark matter (left). The $95 \%$ exclusion contours for by mass of the mediator, $m_{Z^{\prime}}$, and mass of the pseudo scalar, $m_{\mathrm{A}}$ (right) [15]. 
Another Mono-H search for DM is the Mono-H( $\gamma \gamma)$ search with $36.1 \mathrm{fb}^{-1}$ of data [16]. This search has a very clean signature, with the $\gamma \gamma$ non resonant spectrum estimated from sideband regions in data, and SM $H \rightarrow \gamma \gamma$ estimated from MC. Limits on a $Z_{B}^{\prime}$ mediator are placed up to $850 \mathrm{GeV}$ for DM masses up to $200 \mathrm{GeV}$. These are reinterpreted as spin-independent cross section limits and compared to direct detection searches, see Figure 8 . These limits are only competitive at the lowest masses of DM.
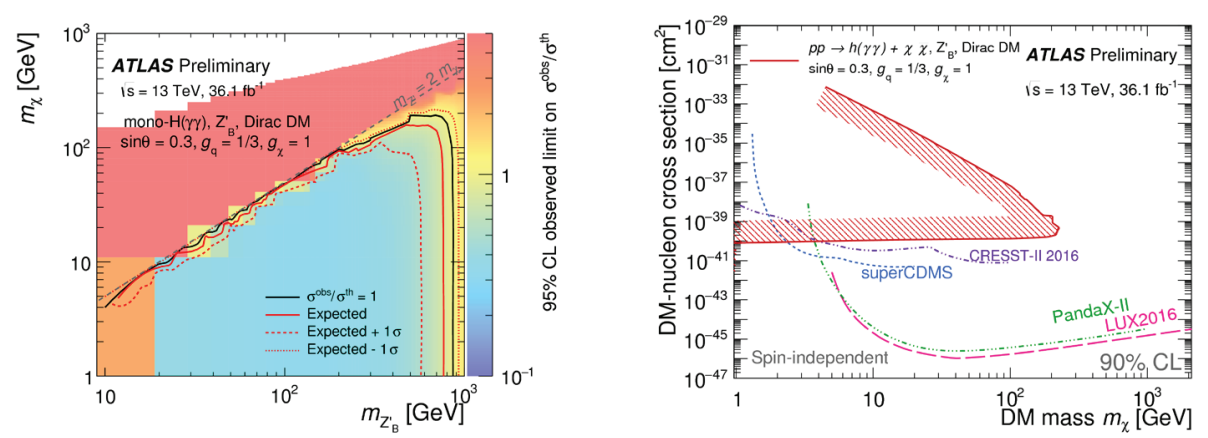

Figure 8. The $95 \%$ exclusion contours for Mono- $\mathrm{H}(\gamma \gamma)$ models of DM, by mass of the mediator, $m_{Z_{B}^{\prime}}$, and mass of the DM, $m_{\chi}$ (left). Exclusion contours at $90 \%$ CL reinterpreted as spin-independent DM-nucleon coupling strengths for various mediator masses (right) [16].

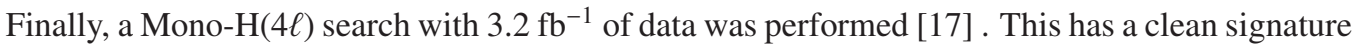
with few background events, which are primarily from SM Higgs and diboson events. Upper limits were set on cross section times branching fraction for models with various masses of mediators and a DM mass of $1 \mathrm{GeV}$, see Figure 9.
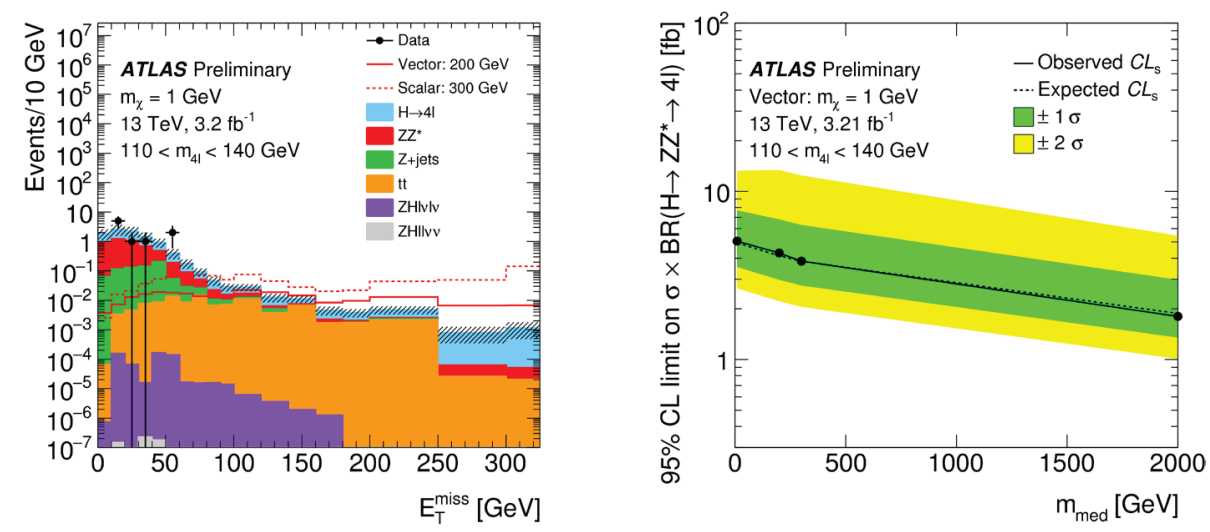

Figure 9. The number of events in the backgrounds to the search as well as the data measurement are shown in bins of $E_{\mathrm{T}}^{\text {miss }}$ up to $350 \mathrm{GeV}$ (left). $95 \% \mathrm{CL}$ limit on branching fraction of vector mediated DM, $m_{\chi}=1 \mathrm{GeV}$ for various mediator masses (right) [17]. 
$E_{T}^{\text {miss }}+$ quarks

The final category of Mono-X searches for DM is from $E_{\mathrm{T}}^{\mathrm{miss}}+$ quarks, in a simplified model such as in Figure 10 (left). This is where a $q \bar{q}$ pair is produced in conjunction with a scalar $(\phi)$ or pseudo scalar $(a)$ mediator, which then decays into DM particles. The searches are being performed with $13.3 \mathrm{fb}^{-1}$ where $q \bar{q}$ can be either $b \bar{b}$ [18] or $t \bar{t}$. In $t \bar{t}$ analyses, the decay products are split by number of leptons $\ell$, and include $0 \ell$ [19], $1 \ell$ [20,21], or $2 \ell$ [22].
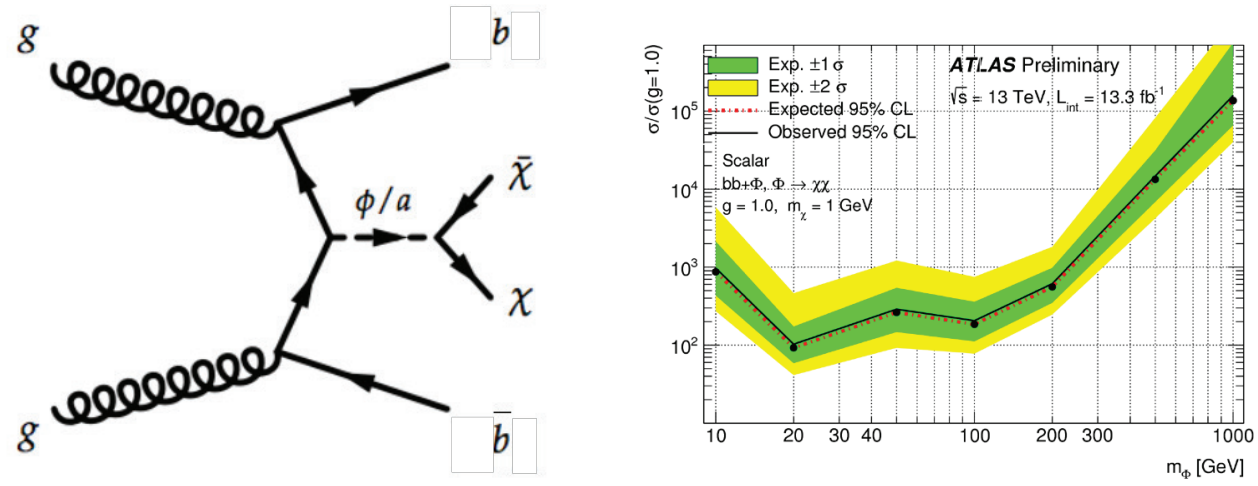

Figure 10. Model of DM produced in conjunction with a $b \bar{b}$ and a scalar or pseudo scalar mediator particle (left) and the limits set on the signal strength of the scalar mediator, for a range of masses (right) [18].

Both the $b \bar{b}$ and $t \bar{t}$ searches look for jets tagged as $b$-jets with between $150 \mathrm{GeV}$ and $350 \mathrm{GeV}$ of $E_{\mathrm{T}}^{\text {miss }}$. Limits are set on the signals strength of the mediator particle (for $b \bar{b}$, see Figure 10 (right)), or in the $m_{\chi}-m_{\text {med }}$ plane up to $\sim 350 \mathrm{GeV}$.

\subsection{Mediator Searches}

If the DM mediator can be produced at the LHC, then it must couple to quarks. Therefore, the mediator should also decay back to SM particles. This will show up as a resonance, such as a $Z^{\prime}$ resonance, in the invariant mass of the decay products. This invariant mass is the parameter of interest for these direct mediator searches, rather than the $E_{\mathrm{T}}^{\mathrm{miss}}$ for the Mono-X searches.

\section{Dijet}

If the mediator couples to quarks, then these would show up in the detector as two energetic jets, known as a dijet event. There are three separate searches for dijet events. The first is a search for at least two jets with a large transverse momentum [23]. This search used $37 \mathrm{fb}^{-1}$ of data, and modelled the background with a fit to a smoothly falling QCD spectrum in $m_{j j}$. It is sensitive in the mass range 1.1-8.2 TeV. Another search with a very similar strategy is the 'Trigger Level Analysis', which probes a lower mass range (0.44-1.2 TeV), as it triggers to record events on a stream of partially rebuilt objects in the detector (tracking and muon information is thrown away), and searched $3.4 \mathrm{fb}^{-1}$ of data [24]. Finally, the dijet + ISR analysis triggers on an event with an energetic photon or jet to look for lower mass dijets, but uses similar background estimations to the first two analyses, and used an intermediate $15.5 \mathrm{fb}^{-1}$ of data to search for DM mediators [25]. This analysis is able to probe the region of $m_{j j}$ in the range $0.17-1.5 \mathrm{GeV}$. 
Each of these analyses uses $y^{*}$, which is half the difference in rapidity of the two jets, to reduce the QCD background. The analyses search ranges of the mass of the mediator $\left(m_{Z^{\prime}}\right)$ for various coupling strengths. The three searches described above are summarized in Figure 11. Finally, not pictured here, a dijet resonance search for $Z^{\prime}$ was done searching exclusively for $t$-quarks [26].

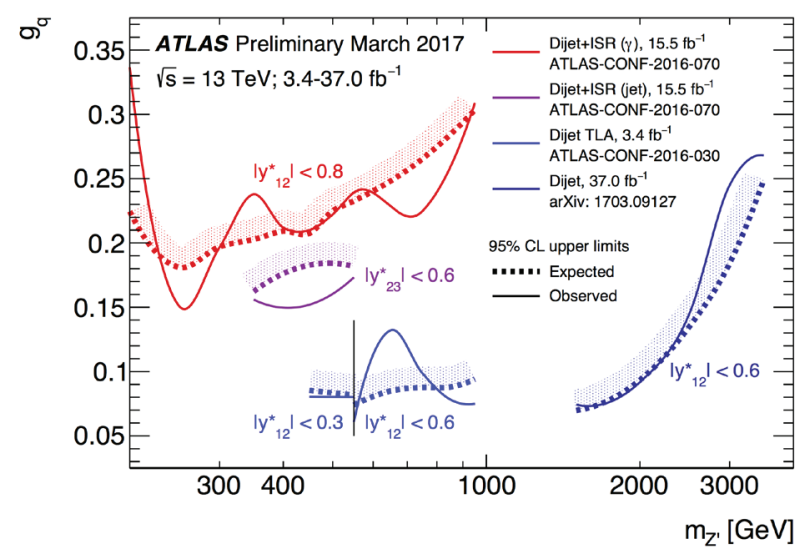

Figure 11. Dijet searches for DM mediator $Z^{\prime}$ for various couplings to quarks $g_{q}$ and masses of $Z^{\prime}$ [23].

\section{Dilepton}

Under the assumption that the mediator also couples to charged leptons, the mediator could decay into pairs of electrons or muons. The search for a mediator decaying to charged leptons $\left(Z^{\prime} \rightarrow \ell \ell\right)$ was done with $36.1 \mathrm{fb}^{-1}$ of data [27]. The main backgrounds from Drell-Yan, $t$-quarks, and dibosons are modelled with MC. Signal regions combined with $e e$ and $\mu \mu$ exclude a mediator with a mass up to 4.1 $\mathrm{TeV}$, see Figure 12.

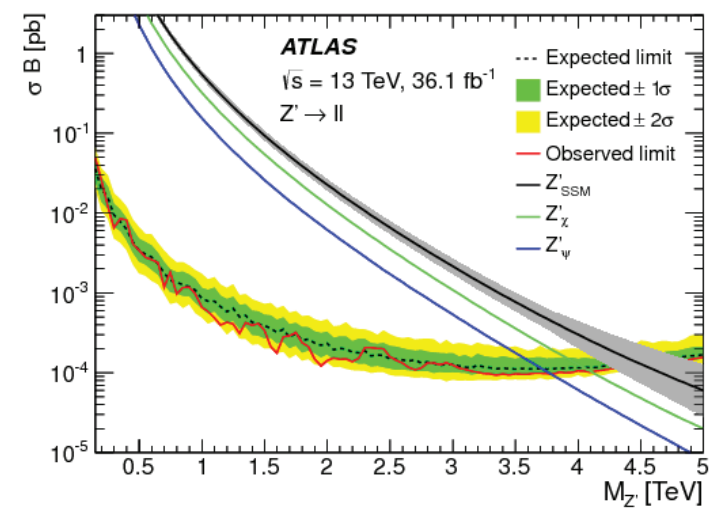

Figure 12. Dilepton exclusion limits on the DM mediator, $Z^{\prime}$ [27]. 


\subsection{Combinations}

A combination of the Mono-X and mediator searches can be seen in Figure 13, [28]. For the simplified models, a common set of parameters from the benchmark models were chosen in order to be able to consistently overlay the results from the different searches.
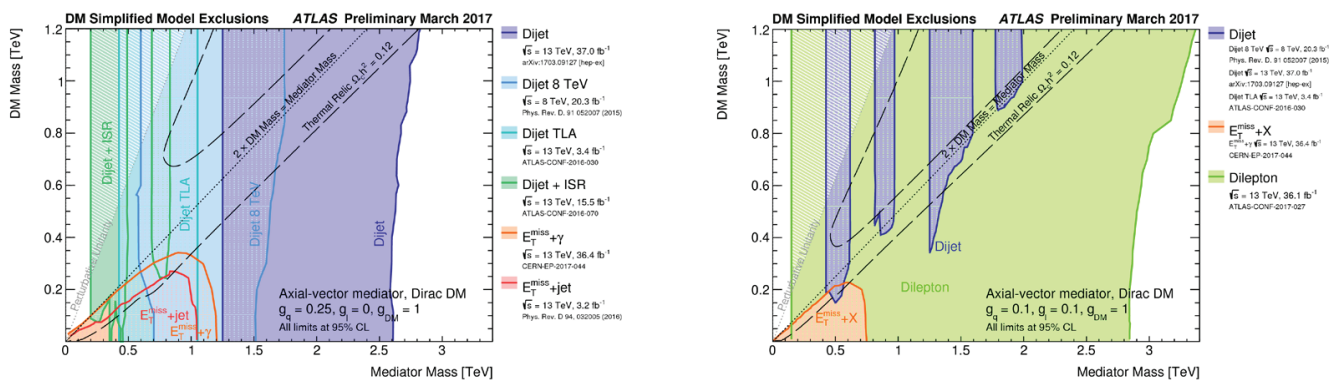

Figure 13. DM Combinations of Mono- $X$ and mediator searches for the axial-vector mediator with no leptonic couplings (left) and small leptonic couplings (right) [28].

These searches can also be reinterpreted on DM-nucleon scattering cross section plots with direct detection searches, under the assumptions that all SM quarks couple equally to the mediator, and that there is only one variant of DM, which couples to this mediator, see Figure 14.
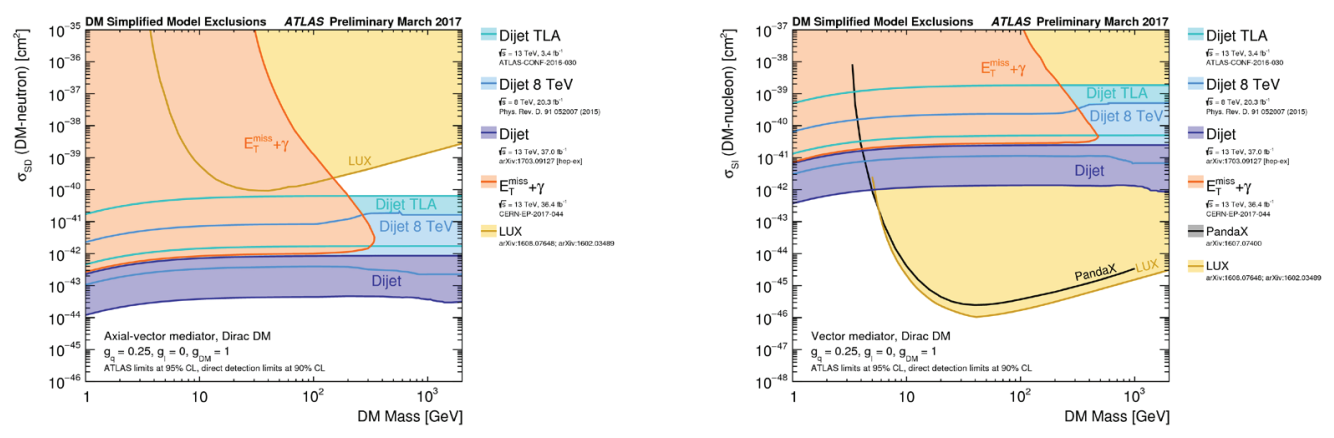

Figure 14. Comparison with direct detection searches with Mono- $X$ and mediator searches for the axial-vector mediator with no leptonic couplings (left) and vector mediator with no leptonic couplings (right) [28].

\subsection{Additional searches}

In addition to the Mono-X and mediator searches presented, there are a large variety of searches at ATLAS to find particle DM. The ones presented previously are not the only ones that are competitive. Supersymmetry (SUSY) searches for the lightest supersymmetric partner, a DM candidate, and their mediators have excellent discrimination power. These searches follow methods similar to those in [29], and set competitive limits on the DM masses of charginos and neutrilinos, the SUSY candidates for DM. 


\section{Summary}

The dark matter searches being performed in ATLAS are complimentary to each other and to direct detection searches. The recommendations have been to move from effective field theories to 'simplified' or mediator models in order to ensure the validity of the models at the large centre-of-mass energy of $13 \mathrm{TeV}$. This has also enabled searches for mediators themselves, providing additional constraints on the dark matter limits being published. There are many interesting $13 \mathrm{TeV}$ results in both 2015 and 2016 data, and many more to come. The results presented here are not exhaustive, but show the cornerstone of the dark matter search program at ATLAS. 


\section{References}

[1] C. Patrignani et al. (Particle Data Group), Chin. Phys. C40, 100001 (2016)

[2] R. Adam et al. (Planck), Astron. Astrophys. 594, A1 (2016)

[3] J.A. Tyson, G.P. Kochanski, I.P. Dell'Antonio, Astrophys. J. 498, L107 (1998)

[4] L. Bergström, Rept. Prog. Phys. 63, 793 (2000)

[5] F. Zwicky, General Relativity and Gravitation 41, 207 (2009)

[6] G. Bertone, D. Hooper, J. Silk, Phys. Rept. 405, 279 (2005)

[7] J.L. Feng, Ann. Rev. Astron. Astrophys. 48, 495 (2010)

[8] ATLAS Collaboration, JINST 3, S08003 (2008)

[9] L. Evans, P. Bryant, Journal of Instrumentation 3, S08001 (2008)

[10] D. Abercrombie et al. (2015), arXiv: 1507.00966 [hep-ex]

[11] ATLAS Collaboration, Eur. Phys. J. C77, 393 (2017)

[12] ATLAS Collaboration, Phys. Rev. D94, 032005 (2016)

[13] ATLAS Collaboration, Phys. Lett. B763, 251 (2016)

[14] ATLAS Collaboration, ATLAS-CONF-2016-056, http://cds . cern. ch/record/2206138

[15] ATLAS Collaboration (2017), arXiv: 1707.01302 [hep-ex]

[16] ATLAS Collaboration (2017), arXiv: 1706.03948[hep-ex]

[17] ATLAS Collaboration, ATLAS-CONF-2015-059, http://cds.cern.ch/record/2114825

[18] ATLAS Collaboration, ATLAS-CONF-2016-086, http://cds.cern.ch/record/2206279

[19] ATLAS Collaboration, ATLAS-CONF-2016-077, http://cds . cern.ch/record/2206250

[20] ATLAS Collaboration, ATLAS-CONF-2016-050, http://cds.cern.ch/record/2206132

[21] ATLAS Collaboration, ATLAS-CONF-2017-037, http://cds. cern. ch/record/2266170

[22] ATLAS Collaboration, ATLAS-CONF-2016-076, http://cds.cern.ch/record/2206249

[23] ATLAS Collaboration (2017), arXiv: 1703.09127 [hep-ex]

[24] ATLAS Collaboration, ATLAS-CONF-2016-030, http://cds.cern.ch/record/2161135

[25] ATLAS Collaboration, ATLAS-CONF-2016-070, http://cds.cern.ch/record/2206221

[26] ATLAS Collaboration, ATLAS-CONF-2016-014, http://cds.cern.ch/record/2141001

[27] ATLAS Collaboration (2017), arXiv: 1707.02424 [hep-ex]

[28] ATLAS Collaboration, Summary plots from the ATLAS Exotic physics group (2017), https: //atlas . web.cern.ch/Atlas/GROUPS/PHYSICS/CombinedSummaryPlots/ EXOTICS/index.html

[29] ATLAS Collaboration, JHEP 09, 175 (2016) 